\title{
Evaluación de los protocolos de aplicación de ácido hialurónico en procesos degenerativos óseos de la articulación temporomandibular. Una revisión de la literatura
}

\section{Application protocol of hyaluronic acid on temporomandibular joint degenerative diseases}

\author{
Coronado L*, Iturriaga $\mathrm{V}^{* *}$, Bornhardt $\mathrm{T} * * *$, Fuentes $\mathrm{R}^{* * * *}$
}

\section{RESUMEN}

Los trastornos temporomandibulares son un grupo heterogéneo de alteraciones funcionales que afectan al aparato masticatorio y reducen la calidad de vida de los pacientes. Dentro de estos trastornos encontramos las alteraciones del complejo cóndilo disco, los cuales son factores de riesgo para la generación de patologías degenerativas articulares de la articulación temporomandibular (ATM). El tratamiento de los procesos degenerativos articulares es controversial ya que se han utilizado a través del tiempo variadas estrategias terapéuticas; desde la utilización de dispositivos ortopédicos mandibulares, pasando por terapias invasivas como la artrocentesis o irrigación y drenaje de los espacios articulares, hasta la infiltración de fármacos dentro del espacio articular. Tradicionalmente la infiltración intra articular de fármacos se ha efectuado con glucocorticoides debido a su efecto antiinflamatorio y analgésico; sin embargo, hace más de dos décadas ha surgido la utilización del ácido hialurónico, cuyo efecto antiinflamatorio y de viscosuplementación ha demostrado poseer gran utilidad terapéutica en procesos degenerativos a nivel articular. Clínicamente, sin embargo, este fármaco carece de un protocolo que forme el esquema terapéutico en cuanto a concentración, dosis, frecuencia y extensión del tratamiento en su uso en patologías degenerativas óseas de la ATM. Por lo tanto, surge la necesidad de revisar la evidencia existente al respecto en estudios experimentales realizados en humanos o animales. Se realizó una revisión de la evidencia disponible online, la que luego de un proceso de análisis y selección obtuvo 11 estudios en humanos y 4 en animales, los autores no encontraron pruebas científicas suficientes para establecer un protocolo de infiltración de ácido hialurónico en patologías degenerativas de la articulación temporomandibular. Palabras clave: Osteoartritis, articulación temporomandibular, ácido hialurónico.

\section{SUMMARY}

Temporomandibular disorders are a heterogeneous group of functional disorders affecting the masticatory system, reducing the quality of life of patients. Within these disorders find the disc condyle complex disorders,

* Estudiante Especialidad y Magíster en Trastornos Temporomandibulares y Dolor Orofacial. Universidad de La Frontera. Temuco, Chile.

** Magíster y Especialista en Trastornos Temporomandibulares y Dolor Orofacial. Policlínico de Trastornos Temporomandibulares y Dolor Orofacial. Facultad Odontología Universidad de La Frontera. Temuco, Chile.

*** Magíster y Especialista en Trastornos Temporomandibulares y Dolor Orofacial. Policlínico de Trastornos Temporomandibulares y Dolor Orofacial. Facultad Odontología Universidad de La Frontera. Temuco, Chile.

**** Doctor med. dent. Centro de investigación Cimofir. Facultad de Odontología. Universidad de La Frontera. Temuco, Chile. 
which are risk factors for the generation of degenerative joint diseases of the temporomandibular joint (TMJ). The treatment of joint degenerative processes is controversial, and have been used over time varied therapeutic strategies, since the use of mandibular orthopedic devices, through invasive therapies such as arthrocentesis or irrigation and drainage of the joint spaces; drugs to infiltration into the joint space. Traditionally the intra infiltration articular drug was performed with glucocorticoids due to its anti-inflammatory and analgesic effect, however more than two decades ago has emerged using the hyaluronic acid, the inflammatory and viscosupplementation effect has demonstrated great therapeutic usefulness in degenerative processes articular level. Clinically this drug however lacks a protocol that regulates the therapeutic regimen in strength, dosage, frequency and extent of treatment in use in bone degenerative diseases of the TMJ. Thus arises the need to review the existing evidence on this in experimental studies in humans or animals. A review of the evidence was made available online, which after a process of analysis and selection scored 11 studies in humans and 4 in animals, the authors found insufficient evidence to establish a protocol infiltration of hyaluronic acid in degenerative pathologies temporo mandibular joint.

Key words: Osteoarthritis, temporomandibular joint, hialuronic acid.

Fecha de recepción: 29 de mayo de 2014.

Aceptado para publicación: 18 de julio de 2014.

Coronado L; Iturriaga V; Bornhardt T, Fuentes R. Evaluación de los protocolos de aplicación de ácido hialurónico en procesos degenerativos óseos de la articulación temporomandibular. Una revisión de la literatura. Av. Odontoestomatol 2015; 31 (2): 77-84.

\section{INTRODUCCIÓN}

Las patologías inflamatorias articulares se producen como resultado de patrones de injuria tiempo dependientes frente a los cuales reaccionan las estructuras articulares con procesos degenerativos (1). Estos cambios degenerativos en el cartílago articular producen síntomas en articulaciones específicas del cuerpo humano. Las articulaciones temporomandibulares (ATM) presentan la particularidad de poseer superficies articulares cubiertas de fibrocartílago a diferencia del cartílago hialino de las demás articulaciones, y pueden ser afectadas por estos procesos degenerativos sufriendo deterioro y abrasión del fibrocartílago articular y de las superficies de los tejidos blandos asociados, además se presenta adelgazamiento y remodelación del hueso subyacente, formación de osteofitos y quistes subcondrales (2). Las principales patologías degenerativas de la ATM son la osteoartritis (OA) y la osteoartrosis (OS), ambas se clasifican según la Asociación Americana de Dolor Orofacial como un trastorno inflamatorio de la ATM clasificada bajo el código 11.7.5 (3).

Los procesos degenerativos de la ATM suelen hallarse más frecuentemente en sujetos de edad avanza- da, los cuales sin embargo frecuentemente no presentan sintomatología dolorosa asociada (osteoartrosis). Estos cambios articulares pueden agudizarse y ser acompañados de sintomatología dolorosa inflamatoria (osteoartritis) y además pueden ser causados o agravados por condiciones articulares generales como la artritis reumatoidea u psoriásica por ejemplo. Es así como el hallazgo a nivel de ATM de OA puede ser la manifestación de enfermedades degenerativas articulares asintomáticas en otros territorios. La sintomatología suele presentarse tardíamente y cuando los cambios patológicos son irreversibles, sin embargo el diagnóstico precoz potencia la efectividad terapéutica y protege las estructuras articulares deteniendo parcialmente la progresión de los procesos degenerativos, disminuyendo la sintomatología y el compromiso funcional articular.

La historia clínica de la OA implica dolor articular unilateral o bilateral constante que se agrava con la función mandibular y frecuentemente de agudización nocturna. Clínicamente, se presenta una limitación dolorosa de la apertura oral, crepitación, dolor funcional articular, dolor a la palpación lateral del cóndilo y a la carga manual aplicada a la articulación; el diagnóstico se puede complementar con apoyo ima- 
genológico (4); a este nivel, la tomografía de ATM (tomografía computarizada de haz cónico y/o tomografía computarizada) y Resonancia Nuclear Magnética (RNM) de ATM (5) nos mostrará signos de alteraciones estructurales del hueso subarticular condilar mandibular o de la fosa articular.

Establecer la prevalencia de la OA de ATM posee dificultades ya que su transcurso asintomático en la mayoría de los casos dificulta su pesquisa clínica, siendo la mayoría de las veces hallazgo radiográfico in vivo y post mortem en estudios histopatológicos. En estudios clínicos se determinan prevalencias de 8-18\%, radiográficos de un $14-44 \%$ e histopatológicos de un 22-84\% (2), de lo anterior podemos inferir que la presencia de OA es dependiente del método de examinación articular, por lo que su diagnóstico y prevalencia se determina generalmente sobre pacientes que se encuentran en tratamiento por otros Trastornos Temporomandibulares (TTM) en su fase aguda (6). Estos estudios han demostrado su mayor ocurrencia en población de edad avanzada y en sexo femenino, sin embargo esta diferencia de género no se hace notoria hasta la quinta década de vida (1).

La estandarización de criterios diagnósticos para la investigación en TTM (Diagnostic Criteria for Temporomandibular Disorders [DC/TMD]) (7) ha permitido la homogeneidad de criterios en la investigación de la prevalencia de los mismos. En el caso de la OA de ATM, ésta es clasificada en el índice I.3.A.2 junto a la osteoartrosis, los hallazgos clínicos considerados en DC/TMD indicativos de esta patología son:

- Historia positiva de, al menos, uno de los siguientes:

1. Ruido articular en los últimos 30 días durante la función o el movimiento mandibular.

2. Reporte del paciente de ruido durante la examinación.

- Al examen clínico:

1. Crépito presente a la palpación durante, al menos, uno de los siguientes movimientos: Apertura, cierre, lateralidad derecha e izquierda o protrusión mandibular.

Además, pueden acompañarse de signos imagenológicos (CBCT, CAT, RNM) de artrosis (aplanamientos, osteofitos, erosiones, quistes subcondrales).
En investigaciones en pacientes consultantes por TTM sometidos a examen clínico y radiográfico mediante tomografía convencional y resonancia magnética, han reportado una prevalencia de 39,5\% de OA en ATM unilateral y 26,3\% bilateral (6), mostrando una mayor ocurrencia que en la población general (8), lo que refuerza a las alteraciones del complejo cóndilo disco como factor de riesgo en la aparición de procesos degenerativos de ATM.

Respecto al tratamiento de la OA, una de las vías terapéuticas son las infiltraciones intraarticulares, las que se han realizado normalmente con Glucocorticoides (CO). Éstas han sido usadas con buenos resultados para el tratamiento de diferentes TTM (913); sin embargo, efectos adversos, como el progreso de la enfermedad articular (12) y reabsorción condilar, se ha descrito en un estudio con animales después de solo una inyección de dexametasona (14).

Por otro lado, la infiltración intraarticular con ácido hialurónico $(\mathrm{AH})$ un polisacárido de alto peso molecular, también ha sido usado en la OA de la ATM, usándose tanto como viscosuplementación como antiinflamatorio (10, 12, 15-19). En pacientes con OA de ATM y/o artritis reumática, el AH fue comparado con glucocorticoides $(10,12)$, no encontrándose diferencia estadísticamente significativa en los resultados de las infiltraciones en ATM. La comparación de ambos medicamentos en OA se ha realizado también en otras articulaciones como en rodilla y de la misma forma no se observó ventajas estadísticamente significativos en favor de uno u otro (20). Otros estudios se han centrado en los efectos positivos del $\mathrm{AH}$ en uso intra articular sin atender a posibles efectos adversos (12, 15-20), salvo un reporte de caso (21).

El uso de $\mathrm{AH}$ en infiltraciones articulares se basa en su efecto de lubricación de las superficies articulares reduciendo la fricción en las cavidades sinoviales lesionadas y que presentan adhesiones, lo que junto a la disminución de factores de la inflamación disminuye el dolor articular $(10,16,22-25)$. Su actividad metabólica facilita la nutrición del disco, cartílago articular y en zonas avasculares. El AH actúa en combinación con glucosaminoglicanos para formar proteoglicanos que bajo condiciones patológicas se desintegran y dispersan en la cavidad sinovial (10, 22-24, 26). Aunque la vida media es de 13 horas, los bene- 
ficios clínicos de su aplicación intraarticular persisten por semanas, incluso meses $(23,26,27)$.

El objetivo de esta revisión fue analizar la evidencia disponible sobre protocolos de aplicación de ácido hialurónico en procesos degenerativos óseos de la ATM.

\section{MATERIAL Y MÉTODO}

Se realizó una búsqueda de las bases de datos electrónicas EBSCO Dentistry and Oral Sciences Source, The Cochrane Library, y MEDLINE-PubMed, Web of Knowledge entre el 1 de enero de 1987 y el 23 de junio del 2013. Los términos de búsqueda utilizados fueron: "Osteoarthritis" (MeSH Terms) AND temporomandibular joint (All Fields) AND ("hyaluronate" [MeSH Terms] OR "hyaluronic acid" [All Fields]). Se restringió los idiomas al español, inglés e italiano.

Se utilizaron los términos booleanos AND para relacionar los términos "osteoarthtritis", "temporomandibular joint" y "hyaluronate"; además, se usó OR para unir a este último con el término "hyaluronic acid".

Dos de los autores analizaron los artículos identificados para determinar su pertinencia al estudio, seleccionando primero por título, resumen y texto completo finalmente.

\section{RESULTADOS}

La búsqueda a través de MEDLINE detallada anteriormente arrojó un resultado de 13 estudios en total; bajo los mismos parámetros The Cochrane Library entregó 6 resultados y la base de datos EBSCO 15. Un total de 15 estudios se seleccionaron, 11 en humanos y 4 en animales. Posteriormente dentro de los estudios seleccionados se analizó el protocolo de infiltración de AH en ATM usado en cada uno de ellos.

\section{Estudios en humanos}

En los 11 estudios revisados y seleccionados realizados en humanos $(10,11,15,16,22,23,27-31)$, se observan diversos esquemas de infiltración de $\mathrm{AH}$, en cuanto a concentración, dosis, frecuencia y extensión del tratamiento. Respecto a la concentración, en 6 estudios se utilizó el AH al 1\% (10, 11, 22, 23, 27-31), en 2 estudios se administró en concentración de 0,5\% (15, 30), y un estudio utilizó AH al 1,5\% (16). En lo concerniente a la dosis, se observan variaciones que van desde los $0,5 \mathrm{ml}$ en 2 estudios (10, 16), $0,7 \mathrm{ml}$ en otros dos (11,31), $1 \mathrm{ml}$ en cuatro (22, $23,28,29)$ y en un caso hasta $2,6 \mathrm{ml}(15)$, finalmente en 2 estudios $(27,30)$ no se detallan las dosis utilizadas. Respecto a la frecuencia, esta va desde infiltraciones únicas $(15,22,27)$, a variadas, dentro de estas últimas: 2 veces por dos semanas $(10,11$, 30, 31), y una infiltración semanal $(16,23,28,29)$. En lo que respecta a la extensión de tratamiento este se encuentra en un rango que va desde la inyección única $(15,22,27)$, extensión de 2 semanas en 4 estudios (10,11, 30, 31), 5 semanas en tres estudios $(23,28,29)$ y finalmente de 6 meses en un estudio (16). Los resultados se detallan en la Tabla 1.

\section{Estudios en animales}

En los cuatro estudios seleccionados, se utilizaron diversas especies animales: conejos $(32,35)$, ratas (33) y ovejas (34), así como distintos métodos para inducir la OA. Respecto al uso de AH; la concentración usada en todos los estudios fue de un $1 \%$, salvo uno (35) donde se utilizó al 0,8 \%. La dosis usadas en los estudios en ratas fue de $0,12 \mathrm{ml}$, en ovejas de $1 \mathrm{ml}$ y en conejos se usó $0,5 \mathrm{ml}$ (35) y 0,1 $\mathrm{ml}$ (32). La frecuencia y extensión del tratamiento en tres estudios fue de infiltraciones únicas (32-34), en cambio se realizaron 3 infiltraciones semanales en un caso (35). Los resultados se muestran en la Tabla 2.

\section{DISCUSIÓN}

La evidencia científica seleccionada y analizada en estudios en humanos muestra la gran variación de esquemas terapéuticos usados en los diversos ensayos clínicos (10, 11, 15, 16, 22, 23, 27-31), manifestando la falta de evidencia existente sobre el mejor protocolo en cuanto a dosis, concentración, frecuencia y extensión del tratamiento en el uso de $\mathrm{AH}$ en 


\begin{tabular}{|c|c|c|c|c|}
\hline \multicolumn{5}{|c|}{ TABLA 1.- ESTUDIOS REVISADOS EN HUMANOS } \\
\hline Autor & Año & Tamaño de muestra & Inyección & Esquema \\
\hline Kopp et al (10) & 1987 & $\begin{array}{l}15 \text { personas } \\
12 \text { personas }\end{array}$ & $\begin{array}{l}\mathrm{AH}(1 \%) \\
\mathrm{CO}\end{array}$ & $0,5 \mathrm{ml}, 2$ veces cada 2 semanas \\
\hline McCain et al (15) & 1989 & $\begin{array}{l}33 \text { ATM } \\
22 \text { ATM }\end{array}$ & $\begin{array}{l}\mathrm{SA}+\mathrm{AH}(0,5 \%) \\
\mathrm{SA}\end{array}$ & $\begin{array}{l}24 \mathrm{ml} \mathrm{SA}+2,6 \mathrm{ml} \mathrm{AH} \\
24 \mathrm{ml} \mathrm{SA}\end{array}$ \\
\hline Kopp et al (11) & 1991 & $\begin{array}{l}14 \text { personas } \\
14 \text { personas } \\
13 \text { personas }\end{array}$ & $\begin{array}{l}\mathrm{AH}(1 \%) \\
\mathrm{CO} \\
\mathrm{SA}\end{array}$ & $0,7 \mathrm{ml}, 2$ veces cada 2 semanas \\
\hline Bertolami et al (27) & 1993 & $\begin{array}{l}80 \text { pacientes } \\
41 \text { pacientes }\end{array}$ & $\begin{array}{l}\mathrm{HA}(1 \%) \\
\mathrm{SA}\end{array}$ & 1 inyección \\
\hline Sato et al $(23,28,29)$ & $\begin{array}{l}1997 \\
2001 \\
2001\end{array}$ & $\begin{array}{l}\text { 26/50 personas } \\
59 / 62 \text { personas } \\
60 / 76 \text { personas }\end{array}$ & $\begin{array}{l}\text { Anestesia } \\
\text { Drenaje + AH (1\%) } \\
\text { Control }\end{array}$ & $\begin{array}{l}1 \mathrm{ml} \text { todas las semanas } \times \\
5 \text { semanas/sin intervención }\end{array}$ \\
\hline Alpaslan et al (22) & 2001 & $\begin{array}{l}23 \text { personas } \\
8 \text { personas }\end{array}$ & $\begin{array}{l}\text { Anestesia, SA + AH } \\
(1 \%) \\
\text { Anestesia, SA }\end{array}$ & $\begin{array}{l}200-300 \mathrm{ml} \text { (artrocentesis) } \\
+1 \mathrm{ml} \text { una vez } \\
200-300 \mathrm{ml} \text { (artrocentesis) }\end{array}$ \\
\hline Hepguler et al (16) & 2002 & $\begin{array}{l}19 \text { personas } \\
19 \text { personas }\end{array}$ & $\begin{array}{l}\mathrm{AH}(1,5 \%) \\
\mathrm{SA}\end{array}$ & $\begin{array}{l}0,5 \mathrm{ml} \text { todas las semanas } \times \\
6 \text { meses }\end{array}$ \\
\hline Bjørnland et al (31) & 2007 & $\begin{array}{l}20 \text { pacientes } \\
20 \text { pacientes }\end{array}$ & $\begin{array}{l}\text { Anestesia + AH (1\%) } \\
\text { Anestesia + CO }\end{array}$ & $\begin{array}{l}2 \text { inyecciones de } 0,7-1 \mathrm{ml} \\
\text { separados } 14 \text { días }\end{array}$ \\
\hline Møystad et al (30) & 2008 & $\begin{array}{l}20 \text { pacientes } \\
20 \text { pacientes }\end{array}$ & $\begin{array}{l}\mathrm{HA}(0,5 \%) \\
\mathrm{CO}\end{array}$ & $\begin{array}{l}2 \text { inyecciones separadas } \times \\
2 \text { semanas }\end{array}$ \\
\hline
\end{tabular}

AH: Ácido hialurónico; CO: Corticoides; SA: Suero salino.

OA de ATM. Además en un estudio (27) no se especifica la dosis de aplicación de AH usada. Los estudios en animales parece el paso previo lógico para lograr establecer el mejor esquema terapéutico de infiltración de $\mathrm{AH}$, sin embargo los estudios realizados en ratas, ovejas, y conejos incluidos en este estudio muestran la misma falta de homogeneidad en la posología de aplicación de AH en ATM en cuanto a dosis, concentración, frecuencia y extensión del tratamiento, encontrando en la literatura revisada 4 esquemas distintos de aplicación de $\mathrm{AH}$.

Los estudios consultados sólo evaluaron los efectos de la infiltración de AH en ATM, o compararon al AH con otros medicamentos, sin embargo en ninguno de los estudios revisados tanto los realizados en hu- manos como los efectuados con animales se realizó una comparación de distintos esquemas terapéuticos de infiltración de AH en OA de la ATM.

Los autores concuerdan con Escoda-Francoli et al (36) quienes, en un estudio, determinaron que la literatura consultada no es suficiente para establecer una posología adecuada de AH en OA de ATM, considerando que un estudio multicéntrico aleatorizado controlado con uso de placebo a doble ciego con una suficiente extensión muestral en grupos bien definidos de TTM es totalmente necesario con el fin de poder establecer los verdaderos efectos terapéuticos de la inyección intra articular de $\mathrm{AH}$ en pacientes con TTM y definir la mejor posología de uso. 


\begin{tabular}{|c|c|c|c|c|c|}
\hline \multicolumn{6}{|c|}{ TABLA 2.- ESTUDIOS REVISADOS EN ANIMALES } \\
\hline Autor & Año & Muestra/inducción & $\begin{array}{c}\text { Tamaño } \\
\text { de muestra }\end{array}$ & Inyección & Esquema \\
\hline $\begin{array}{l}\text { Chang-Hwan } \\
\text { et al (34) }\end{array}$ & 2001 & 10 ovejas/quirúrgica & $\begin{array}{c}6 \\
2 \text { (sin inducción) } \\
2 \text { (sin inducción) }\end{array}$ & $\begin{array}{l}1 \mathrm{ml} \mathrm{AH} 1 \% \text { en ATM izq. } \\
1 \mathrm{ml} \mathrm{SA} \text { en ATM der. } \\
1 \mathrm{ml} \mathrm{SA} \text { ambas ATM. }\end{array}$ & $\begin{array}{l}\text { Infiltración a los } 7 \text {, } \\
10,14,17,21 \text { días }\end{array}$ \\
\hline Yin et al (32) & 2005 & 25 conejos/enzimática & $\begin{array}{c}1 \text { (control) } \\
8 \\
8 \\
8\end{array}$ & $\begin{array}{l}\quad- \\
\text { Artrocentesis } \\
\text { AH } 0,1 \mathrm{ml} \text { al } 1 \% \\
\text { Artrocentesis }+A H \\
0,1 \mathrm{ml} \text { al } 1 \%\end{array}$ & $\begin{array}{l}\quad- \\
\text { Solo se trató ATM } \\
\text { izq. realizando } \\
\text { inyección } 1 \mathrm{vez} \\
\text { por semana } \\
\text { por } 3 \text { semanas, } \\
\text { ATM dcha. se usó } \\
\text { como control. }\end{array}$ \\
\hline $\begin{array}{l}\text { Ibrahim E. } \\
\text { et al (33) }\end{array}$ & 2011 & 24 ratas/mecánica & $\begin{array}{c}6 \text { (controles sin tto.) } \\
18 \text { tratadas }\end{array}$ & $\begin{array}{l}-- \\
0,12 \mathrm{ml} \text { AH } 1 \% \text { ATM dcha. } \\
0,12 \text { ml SA ATM izq. }\end{array}$ & 1 sola vez \\
\hline $\begin{array}{l}\text { Duygu et } \\
\text { al (35) }\end{array}$ & 2011 & $\begin{array}{l}24 \text { conejos/mono } \\
\text { iodo acetato }\end{array}$ & $\begin{array}{l}24 \text { ATM dcha. } \\
24 \text { ATM izq. }\end{array}$ & $\begin{array}{l}0,5 \mathrm{ml} \mathrm{AH} 0,8 \% \\
0,5 \mathrm{ml} \mathrm{SA}\end{array}$ & $\begin{array}{l}\text { Una vez semanal } \\
\text { por } 3 \text { semanas }\end{array}$ \\
\hline
\end{tabular}

AH: Ácido hialurónico; SA: Suero salino.

La realización de ensayos clínicos aleatorizados en animales que comparen los efectos de diversos esquemas de infiltración de AH en ATM surge como una necesidad de establecer evidencia que avale y fundamente la elaboración de un protocolo científico de aplicación clínica de AH en ATM de seres humanos.

\section{BIBLIOGRAFÍA}

1. Zarb G, Carlsson G. Temporomandibular disorders: osteoarthritis. J Orofac Pain 1999;13: 295-306.

2. Grandmont P. Osteoarthrosis/osteoarthritis in the temporomandibular joints. Int $\mathrm{J}$ Prosthodont 2009;22:530-2.

3. de Leeuw R. Orofacial Pain: Guidelines for Assessment, Diagnosis, and Management. Quintessence Pub Co: Barcelona; 2008.
4. Okeson JP. Tratamiento de oclusión y afecciones temporomandibulares. Elsevier: Barcelona; 2008.

5. López López J, Chimenos Küstner E, Blanco Carrión A, Reselló Llabrés X, Jané Salass E. Diagnóstico por la imagen de los trastornos de la articulación craneomandibular. Av Odontoestomatol 2005;21:71-88.

6. Manfredini D, Chiappe G, Bosco M. Research diagnostic criteria for temporomandibular disorders (RDC/TMD) axis I diagnoses in an Italian patient population. J Oral Rehabil 2006;33:5518.

7. Schiffman E, Ohrbach R, Truelove E, Look J, Anderson G, Jensen R, et al. Diagnostic Criteria for Temporomandibular Disorders (DC/ TMD) for Clinical and Research Applications: recommendations of the International RDC/TMD Consortium Network* and Orofacial Pain Special 
Interest Group †. J Oral Facial Pain Headache 2014;28:6-27.

8. Rantala MA, Ahlberg J, Suvinen TI, Savolainen A, Könönen M. Symptoms, signs, and clinical diagnoses according to the research diagnostic criteria for temporomandibular disorders among Finnish multiprofessional media personnel. J Orofac Pain 2003;17:311-6.

9. Kopp S, Wenneberg B. Effects of occlusal treatment and intraarticular injections on temporomandibular joint pain and dysfunction. Acta Odontol Scand 1981;39:87-96.

10. Kopp S, Carlsson GE, Haraldson T, Wenneberg B. Long-term effect of intra-articular injections of sodium hyaluronate and corticosteroid on temporomandibular joint arthritis. J Oral Maxillofac Surg 1987;45:929-35.

11. Kopp S, Akerman S, Nilner M. Short-term effects of intraarticular sodium hyaluronate, glucocorticoid, and saline injections on rheumatoid arthritis of the temporomandibular joint. J Craniomandib Disord 1991;5:231-8.

12. Wenneberg B, Kopp S, Gröndahl HG. Long-term effect of intra-articular injections of a glucocorticosteroid into the TMJ: a clinical and radiographic 8-year follow-up. J Craniomandib Disord 1991;5:11-8.

13. Alstergren P, Appelgren A, Appelgren B, Kopp S, Lundeberg $T$, Theodorsson $E$. The effect on joint fluid concentration of neuropeptide $\mathrm{Y}$ by intraarticular injection of glucocorticoid in temporomandibular joint arthritis. Acta Odontol Scand 1996;54:1-7.

14. El-Hakim IE, Abdel-Hamid IS, Bader A. Temporomandibular joint (TMJ) response to intraarticular dexamethasone injection following mechanical arthropathy: a histological study in rats. Int J Oral Maxillofac Surg 2005;34:30510.

15. McCain JP, Balazs EA, de la Rua H. Preliminary studies on the use of a viscoelastic solution in arthroscopic surgery of the temporomandibular joint. J Oral Maxillofac Surg 1989;47:1161-8.

16. Hepguler S, Akkoc YS, Pehlivan M, Ozturk C, Celebi G, Saracoglu A, et al. The efficacy of intra-articular sodium hyaluronate in patients with reducing displaced disc of the temporomandibular joint. J Oral Rehabil 2002;29:80-6.

17. Guarda-Nardini L, Tito R, Staffieri A, Beltrame A. Treatment of patients with arthrosis of the temporomandibular joint by infiltration of sodium hyaluronate: a preliminary study. Eur Arch Otorhinolaryngol 2002;259:279-84.

18. Guarda-Nardini L, Masiero S, Marioni G. Conservative treatment of temporomandibular joint osteoarthrosis: intraarticular injection of sodium hyaluronate. J Oral Rehabil 2005;32: 729-34.

19. Yeung RW, Chow RL, Samman N, Chiu K. Shortterm therapeutic outcome of intra-articular high molecular weight hyaluronic acid injection for nonreducing disc displacement of the temporomandibular joint. Oral Surg Oral Med Oral Pathol Oral Radiol Endod 2006;102:453-61.

20. Leopold SS, Redd BB, Warme WJ, Wehrle PA, Pettis PD, Shott S. Corticosteriod compared with hyaluronic acid injections for the treatment of osteoarthritis of the knee. A prospective, randomized trial. J Bone Joint Surg Am 2003;85A:1197-203.

21. lida K, Kurita K, Tange K, Yoshida K. Necrosis of the articular tubercle after repeated injections of sodium hyaluronate in the temporomandibular joint. A case report. Int J Oral Maxillofac Surg $1998 ; 27: 278-9$

22. Alpaslan GH, Alpaslan C. Efficacy of temporomandibular joint arthrocentesis with and without injection of sodium hyaluronate in treatment of internal derangements. J Oral Maxillofac Surg 2001;59:613-8.

23. Sato S, Oguri S, Yamaguchi K, Kawamura H, Motegi K. Pumping injection of sodium 
hyaluronate for patients with non-reducing disc displacement of the temporomandibular joint: two year follow-up. J Craniomaxillofac Surg 2001; 29:89-93.

24. Alpaslan C, Bilgihan A, Alpaslan GH, Güner B, Ozgür Yis M, Erbaş D. Effect of arthrocentesis and sodium hyaluronate injection on nitrite, nitrate, and thiobarbituric acid-reactive substance levels in the synovial fluid. Oral Surg Oral Med Oral Pathol Oral Radiol Endod 2000;89:686-90.

25. Sato S, Sakamoto M, Kawamura H, Motegi K. Disc position and morphology in patients with nonreducing disc displacement treated by injection of sodium hyaluronate. Int $\mathrm{J}$ Oral Maxillofac Surg 1999;28:253-7.

26. Shi Z, Guo C, Awad M. Hyaluronate for temporomandibular joint disorders. Cochrane Database Syst Rev 2013;10:CD002970.

27. Bertolami CN, Gay T, Clark GT, Rendell J, Shetty $\mathrm{V}$, Liu C, et al. Use of sodium hyaluronate in treating temporomandibular joint disorders: a randomized, double-blind, placebo-controlled clinical trial. J Oral Maxillofac Surg 1993;51:232-42.

28. Sato S, Goto S, Kasahara T, Kawamura H, Motegi K. Effect of pumping with injection of sodium hyaluronate and the other factors related to outcome in patients with non-reducing disk displacement of the temporomandibular joint. Int J Oral Maxillofac Surg 2001;30:194-8.

29. Sato S, Ohta M, Ohki H, Kawamura H, Motegi K. Effect of lavage with injection of sodium hyaluronate for patients with nonreducing disk displacement of the temporomandibular joint. Oral Surg Oral Med Oral Pathol Oral Radiol Endod 1997;84:241-4.

30. Møystad A, Mork-Knutsen BB, Bjørnland T. Injection of sodium hyaluronate compared to a corticosteroid in the treatment of patients with temporomandibular joint osteoarthritis: a CT evaluation. Oral Surg Oral Med Oral Pathol Oral Radiol Endod 2008;105:e53-60.
31. Bjørnland T, Gjaerum AA, Møystad A. Osteoarthritis of the temporomandibular joint: an evaluation of the effects and complications of corticosteroid injection compared with injection with sodium hyaluronate. J Oral Rehabil 2007;34: 583-9.

32. Xinmin Y, Jian H. Treatment of temporomandibular joint osteoarthritis with viscosupplementation and arthrocentesis on rabbit model. Oral Surg Oral Med Oral Pathol Oral Radiol Endod 2005; 100:e35-8.

33. El-Hakim IE, Elyamani AO. Preliminary evaluation of histological changes found in a mechanical arthropatic temporomandibular joint (TMJ) exposed to an intra-articular Hyaluronic acid (HA) injection, in a rat model. J Craniomaxillofac Surg 2011;39;610-4.

34. Kim CH, Lee BJ, Yoon J, Seo KM, Park JH, Lee JW, et al. Therapeutic effect of hyaluronic acid on experimental osteoarthrosis of ovine temporomandibular joint, J Vet Med Sci 2001;63: 1083-9.

35. Duygu G, Güler $N$, Cam B, Kürkçü $M$. The effects of high molecular weight hyaluronic acid (Hylan G-F 20) on experimentally induced temporomandibular joint osteoartrosis: part II. Int J Oral Maxillofac. Surg 2011;40:1406-13.

36. Escoda-Francolí J, Vázquez-Delgado E, Gay-Escoda C. Scientific evidence on the usefulness of intraarticular hyaluronic acid injection in the management of temporomandibular dysfunction. Med Oral Patol Oral Cir Bucal 2010;15:e644-8.

\section{CORRESPONDENCIA}

Ramón Fuentes

Facultad Odontología

Universidad de La Frontera

Manuel Montt, 115

Temuco. Chile

E-mail: ramón.fuentes@ufrontera.cl 